\title{
Efficacy and Safety of Alogliptin in Elderly Patients With Type 2 Diabetes Mellitus
}

\author{
Hiroshi Takeda ${ }^{a}$, Nobuo Sasai ${ }^{a}$, Shogo Ito ${ }^{a}$, Mitsuo Obana ${ }^{a}$, Tetsuo Takuma ${ }^{a}$, \\ Masahiko Takai ${ }^{a}$, Hideaki Kaneshige ${ }^{a}$, Hideo Machimura ${ }^{a}$, \\ Akira Kanamori $^{\mathrm{a}}$, Ikuro Matsuba ${ }^{\mathrm{a}, \mathrm{b}}$
}

\begin{abstract}
Background: In Japan, with increasing age of the population, diabetic patients often become in need of hemodialysis due to diabetic nephropathy, and thus there is a demand for development of diabetic treatments that take into account renal effects in the elderly. No previous studies of alogliptin had focused on Japanese elderly subjects; we therefore assessed the effects of alogliptin in elderly individuals using available data.
\end{abstract}

Methods: Laboratory data were compiled for 1 year at intervals of 3 months following the start of alogliptin treatment. The subjects were divided into three groups by age: $<65$ years $(\mathrm{n}=110), 65-74$ years $(n=87)$, and $\geq 75$ years $(n=93)$. Laboratory values in comparison with baseline were compared within groups at various time points, and changes from baseline were compared among the different groups.

Results: Hemoglobin A1c (HbA1c) levels decreased significantly from baseline values in all groups at and after month 3: the change at month 12 was $-0.74 \pm 1.45 \%$ for the age group $<65,-0.47 \pm 1.02 \%$ for the age group $65-74$, and $-0.42 \pm 1.11 \%$ for the age group $\geq 75$. The 12 -month change in estimated glomerular filtration rate (eGFR) was $-6.5 \pm 12.0$ for the age group $<65,-2.0 \pm 8.4$ for the age group $65-$ 74 , and $-1.5 \pm 10.0$ for the age group $\geq 75$; the reduction in the age group $<65$ was significant, whereas the reduction in the age groups $\geq 65$ was not.

Conclusions: Alogliptin significantly lowers HbAlc levels in the elderly and can be used without posing any safety issues, including renal effects, thus contributing to safe blood glucose control in clinical practice.

Keywords: Type 2 diabetes; Dipeptidyl peptidase-4 inhibitor; Alogliptin; eGFR; Elderly

Manuscript submitted August 14, 2019, accepted August 23, 2019

aDiabetes Committee Study Group, Kanagawa Physicians Association, Kanagawa, Japan

${ }^{b}$ Corresponding Author: Ikuro Matsuba, Kanagawa Diabetes Task Force, Kanagawa Physicians Association, 1-3 Fujimichou, Nakaku, Yokohama, Kanagawa 231-0037, Japan. Email: ikuro@matsuba-web.com

doi: https://doi.org/10.14740/jocmr3953

\section{Introduction}

According to a statement of the Japanese Society for Dialysis Therapy, the total number of patients undergoing prolonged hemodialysis therapy in Japan was 334,505 as of the end of 2017 , representing an increase of 4,896 patients compared with the previous year [1]. The most common primary disease affecting the increasing number of hemodialysis patients was diabetic nephropathy, which accounted for $45.6 \%$ of male patients and $35.8 \%$ of female patients. The mean age of all hemodialysis patients was 68.9 years for male patients and 71.4 years for female patients. A stratified analysis by 5 -year age span showed the most common age group to be $75-79$ years for male patients and 80 - 84 years for female patients. While attention should be paid to the effects of drug treatments and drug-induced renal disorders [2], the aforementioned background places importance to diabetic treatments in elderly Japanese patients, with renal effects in mind.

Dipeptidyl peptidase-4 (DPP-4) inhibitors selectively inhibit DPP-4, an enzyme that inactivates incretin in living organisms, to raise blood incretin concentrations and to stimulate glucose-dependent insulin secretion [3]. Many DPP-4 inhibitors have persistent effects when administered once daily, and hypoglycemia and body weight (BW) changes are unlikely [4]; they have been used commonly in Japan since their approval.

We previously reported the efficacy and safety of the DPP4 inhibitor alogliptin used in 1-year treatment [5]. Many studies have so far reported on alogliptin, including the EXAMINE study, which reported that alogliptin treatment did not increase the mortality rate due to cardiovascular events or the hospitalization rate due to heart failure [6], and another study reporting that alogliptin significantly reduced the cardiovascular deaths and all-cause mortality rates in some populations [7]. In addition to clinical trial data, many studies in Japanese subjects reported the efficacy and safety of alogliptin [8-10], including a study reporting that alogliptin treatment lessened the progression of carotid atherosclerosis in terms of intima media thickness (IMT) in type 2 diabetic patients with no cardiovascular disease compared with conventional treatments [11] and good blood glucose control achieved even in long-term observation periods exceeding 3 years [12].

To assess the effects of alogliptin in elderly patients, Pratley et al conducted a meta-analysis of the results from six studies, reporting that alogliptin improved hemoglobin A1c 
(HbAlc) to extents similar to those in young patients, with no increased risks of hypoglycemia, BW gains, or other parameters compared with the young patients [13]. In addition, Rosenstock et al compared the 1-year effects of alogliptin and glipizide in elderly patients, reporting that alogliptin maintained blood glucose control equivalent to that with glipizide, with a much lower risk of hypoglycemia and no BW gain [14]. No study has focused on elderly Japanese patients, however; the meta-analysis by Pratley et al did not include Japanese clinical study results, and the study by Rosenstock et al reported US data only.

With this background, we analyzed compiled data for patients receiving alogliptin in an exploratory manner and assessed its efficacy and safety in elderly patients with type 2 diabetes $(\geq 65$ years).

\section{Patients and Methods}

\section{Study design}

A multicenter retrospective observational study was conducted at clinics and hospitals belonging to the Kanagawa Physicians Association. Data were collected from the medical records of the subjects and the follow-up period was 1 year. This study was approved by the Ethics Review Board of the Kanagawa Physicians Association (May 23, 2013) (approval number: 2521).

\section{Patients}

Patients were eligible for this study if they had type 2 diabetes mellitus (T2DM), were aged 20 years old or older, regularly attended a clinic or hospital belonging to the Kanagawa Physicians Association, and received treatment with alogliptin for 1 year or longer. Alogliptin was started if glycemic control was inadequate for at least 1 month despite diet and exercise therapy or diet and exercise plus oral antidiabetic drugs.

The exclusion criteria were as follows: a history of hypersensitivity to any component of alogliptin; a history of severe ketoacidosis, diabetic coma or precoma within 6 months before the start of alogliptin therapy; severe infection; recent or planned surgery or severe trauma; concurrent use of insulin preparations or glinides; and patients who the attending doctor considered to be inappropriate for this study for other reasons.

\section{Items investigated}

The baseline characteristics investigated for the subjects included the gender, age, height, duration of diabetes, family history, smoking history, alcohol history, and complications. Use of the following drugs was assessed before treatment with alogliptin, at the start of treatment, and 12 months after the start of treatment: alogliptin and other antidiabetic drugs. Efficacy endpoints were determined at each of the specified times, including HbAlc (National Glycohemoglobin Stand- ardization Program value), blood glucose (fasting and postprandial), BW, body mass index (BMI), blood pressure (BP; systolic/diastolic), liver function parameters (glutamate oxaloacetate transaminase, glutamate pyruvate transaminase, and $\gamma$-glutamyl transpeptidase), kidney function parameters (serum creatinine, estimated glomerular filtration rate (eGFR)), serum lipids (total cholesterol (TC), low-density lipoprotein cholesterol (LDL-C), high-density lipoprotein cholesterol (HDL-C), triglycerides (TGs)), and serum amylase. In addition, adverse events were evaluated at each of these times to assess safety.

\section{Statistical analysis}

After patients who did not receive the study drug were excluded from those whose case records were collected, the remaining patients formed the safety analysis set. The full analysis set (FAS) was obtained by excluding patients who met any of the following criteria from the safety analysis set: 1) No HbA1c data at the start of alogliptin treatment; 2) No HbA1c data after the start of alogliptin treatment; 3) Administration of another DPP-4 inhibitor after the start of alogliptin treatment; and 4) Patients for whom age data were not available.

Data were statistically analyzed in three age groups: $<65$ years, $65-74$ years, and $\geq 75$ years.

Patient characteristics were compared using analysis of variance (ANOVA) for continuous variables and Chi-square test for nominal variables. With regard to the efficacy in each of the three groups, a paired $t$-test was used to evaluate the changes in various parameters from baseline at months 3,6 , 9 , and 12, and ANOVA was used for intergroup comparisons. With regard to eGFR, changes from month 3 values at months 6,9 , and 12 after treatment initiation were also evaluated by paired $t$-test. In all analyses, two populations were available: all subjects and subjects newly receiving alogliptin. Intragroup comparisons did not take multiplicity into account, and the significance level was set at 5\% (two-sided). The analytical software R version 3.4.1 (R Foundation for Statistical Computing, Vienna, Austria (https://www.R-project.org/)) was used for calculations. Data are presented as mean \pm standard deviation or number (proportion) of patients.

\section{Results}

Of the 330 patients enrolled in the study 290 who met the selection criteria and did not meet any of the exclusion criteria were selected for the subanalysis set (Fig. 1). Study population breakdown by age group was as follows: 110 patients $(37.9 \%)$ at $<65$ years, 87 patients $(30.0 \%)$ at $65-74$ years, and 93 patients at $\geq 75$ years $(32.1 \%)$. The proportion of women and disease duration increased with increasing age, and the proportion of patients with hepatic steatosis, drinking history, or family history increased with younger age (Table 1). Similar tendencies were noted in the patients newly receiving alogliptin; however, there was no significant difference in sex ratio or incidence of hepatic steatosis in those patients (Supplementary Table 1, www.jocmr.org). 


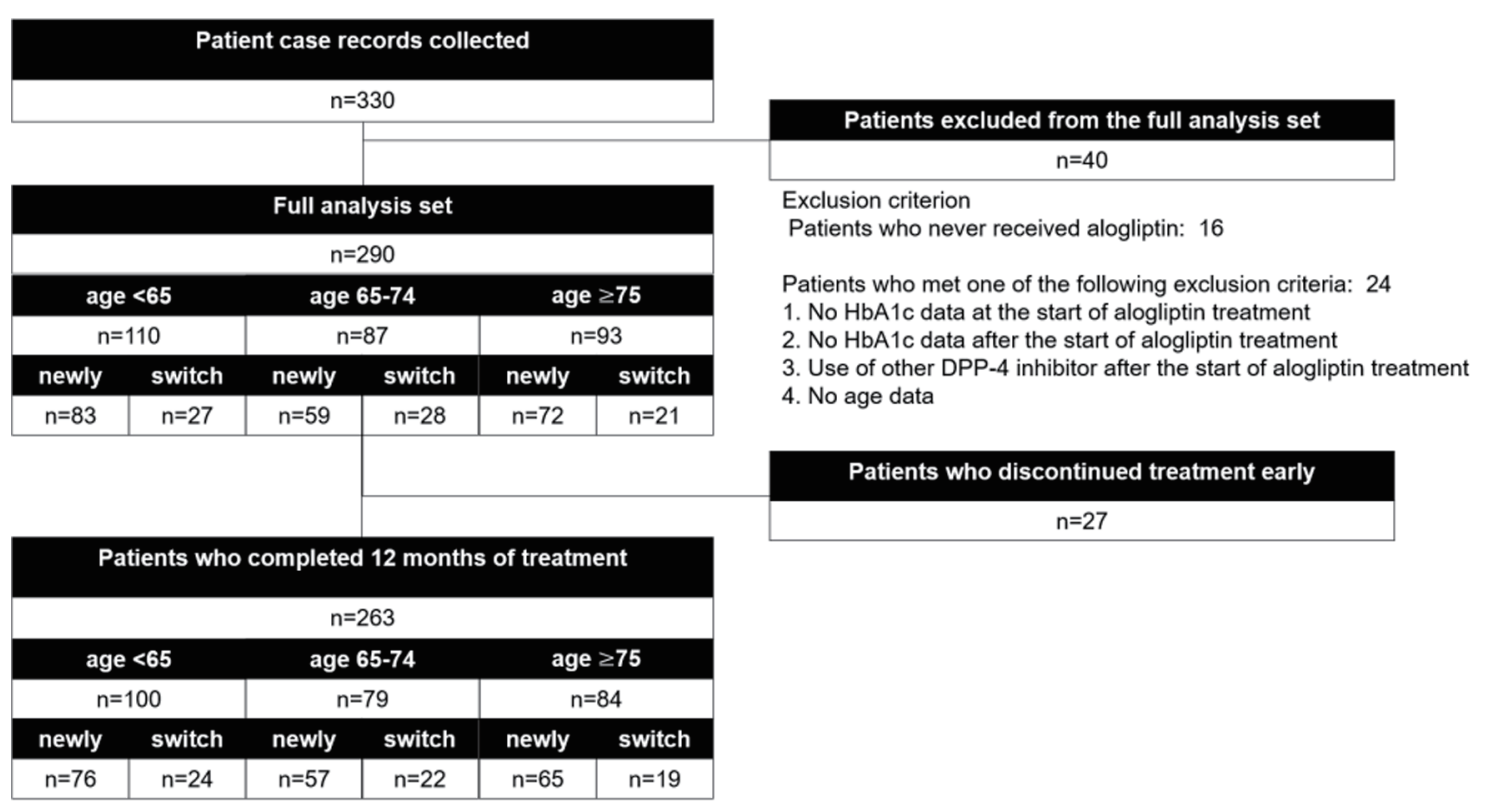

Figure 1. Participant flow.

There was no significant difference in the proportion of patients who were switched from other DPP-4 inhibitors among the three groups (27 patients, $24.5 \%$, at $<65$ years; 28 patients, $32.2 \%$, at $65-74$ years; and 21 patients, $22.6 \%$, at $\geq 75$ years); however, concomitant use of biguanide (BG) decreased with increasing age (Table 2). The same tendency was found in the patients newly receiving alogliptin (Supplementary Table 2, www.jocmr.org).

Changes in eGFR are shown in Figure 2. In the age group $<65$, eGFR decreased significantly from the baseline value of $89.0 \pm 20.8$ at all time points. At and after month 3 , there was no significant change from month 3 value $(3-6$ months change $-0.8 \pm 9.5, \mathrm{P}=0.536 ; 3-9$ months change $-0.3 \pm 10.6$, $\mathrm{P}=0.817$; and $3-12$ months change $-1.7 \pm 11.4, \mathrm{P}=0.232$ ). In the age group 65 - 74, eGFR tended to decrease without significant change. In the age group $\geq 75$, a significant decrease $(-2.9 \pm 9.4)$ was noted only at month 3 ; however, no significant change was noted after that time. The analysis results from the patients newly receiving alogliptin were similar to those from all patients; however, there was no significant intragroup difference in change from baseline in the age group $<65$ at month 3 , with intergroup differences in change noted only at month 9 and month 12 (Fig. 3).

Changes in other parameters are shown in Table 3. HbA1c levels decreased significantly in all age groups at all measuring time points: the change at month 12 was $-0.74 \pm 1.45 \%$ for the age group $<65,-0.47 \pm 1.02 \%$ for the age group $65-74$, and $-0.42 \pm 1.11 \%$ for the age group $\geq 75$. Significant differences in change were noted among the age groups only at month 3 ; however, after that time and until month 12 , no difference was noted among the age groups. In the age group $<65$, the percentage of BG users significantly increased at month 12 compared to the baseline ( $\mathrm{P}=0.033$, Chi-square test). However, in the patients who belong to the age group $<65$ and who did not newly begin using $\mathrm{BG}, \mathrm{HbA} 1 \mathrm{c}$ decreased significantly from the baseline value of $7.46 \pm 1.41$ to month 12 value of $6.78 \pm$ 1.17 ( $\mathrm{P}<0.001$ paired $t$-test). Analysis of the patients newly receiving alogliptin also revealed change differences among the age groups at month 6 ; however, there were similar changes at other time points (Supplementary Table 3, www.jocmr. org). With regard to changes in blood pressure, body weight, and BMI, there was no significant difference among the various age groups. Although some other parameters showed intergroup differences in change, none of them prevailed throughout the entire study period.

Hypoglycemia occurred in one patient in the age group $<65$ and one patient in the age group $65-74$; constipation in three patients in the age group $\geq 75$; neurological disease, hypertension, and dyslipidemia each in one patient in the age group $<65$; and bone fracture in one patient in the age group $\geq 75$.

\section{Discussion}

The present study demonstrated that alogliptin was safe and effective in elderly Japanese patients. Even though serum amylase and creatinine were elevated for month 3 and month 6 after the administration, there were no cases that reported an adverse event.

eGFR decreased significantly in the age group $<65$ at month 3, after which time, however, the level remained constant, with no further reductions. The baseline eGFR for the age group $<65$ was somewhat high, at $89.0 \pm 20.8$. Increased eGFR also represents an unwanted condition, with excessive filtration due to hyperglycemia, suggesting a reduction in eGFR and a restoration of normal state. In the age group $\geq$ 75 , eGFR decreased significantly by $-2.9 \pm 9.4$ at month 3 . However, at and after month 6 , there was no significant difference, and the 12-month change was $-1.5 \pm 10.0$; the signifi- 


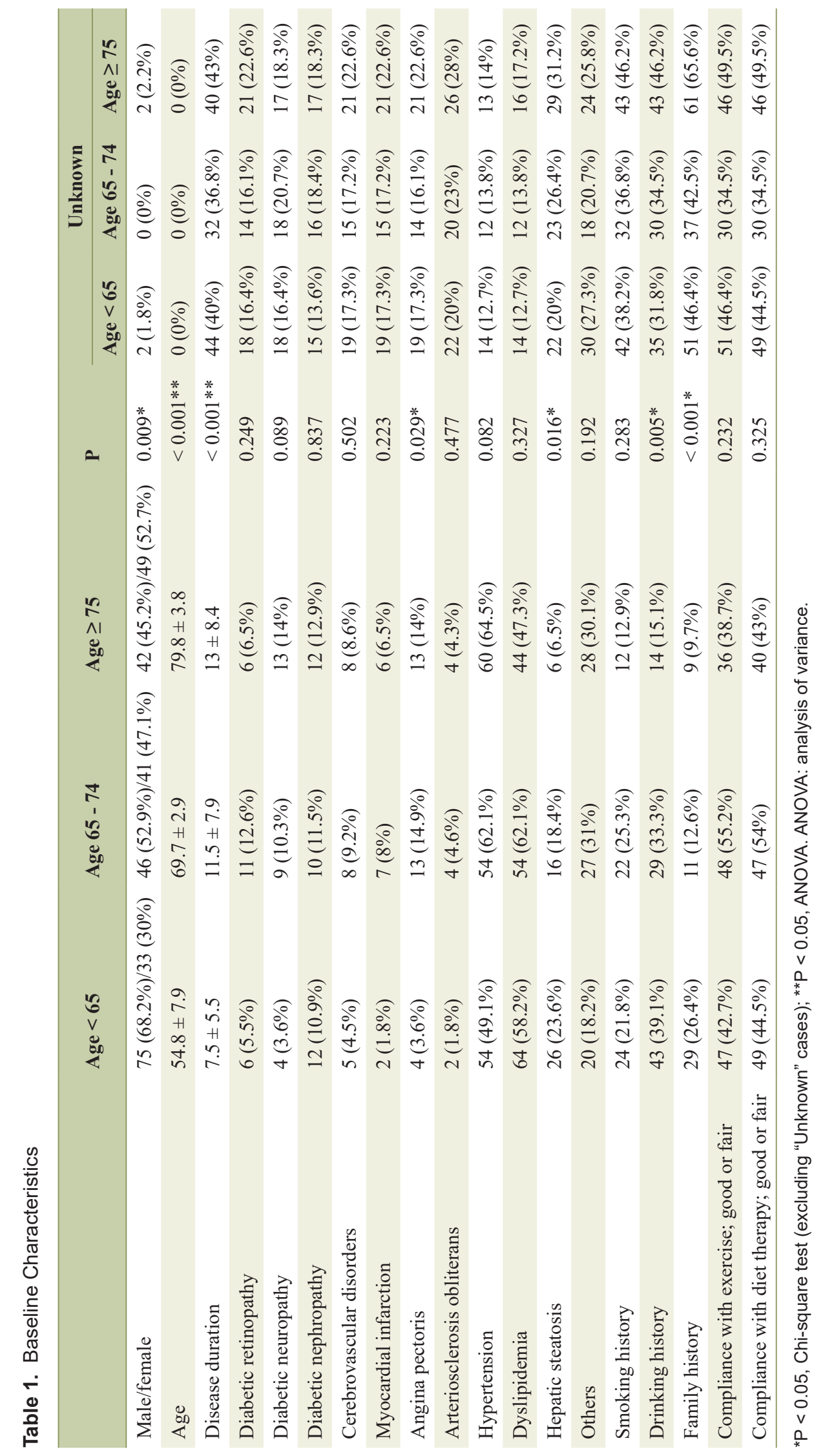


Table 2. Medications of the Full Analysis Set

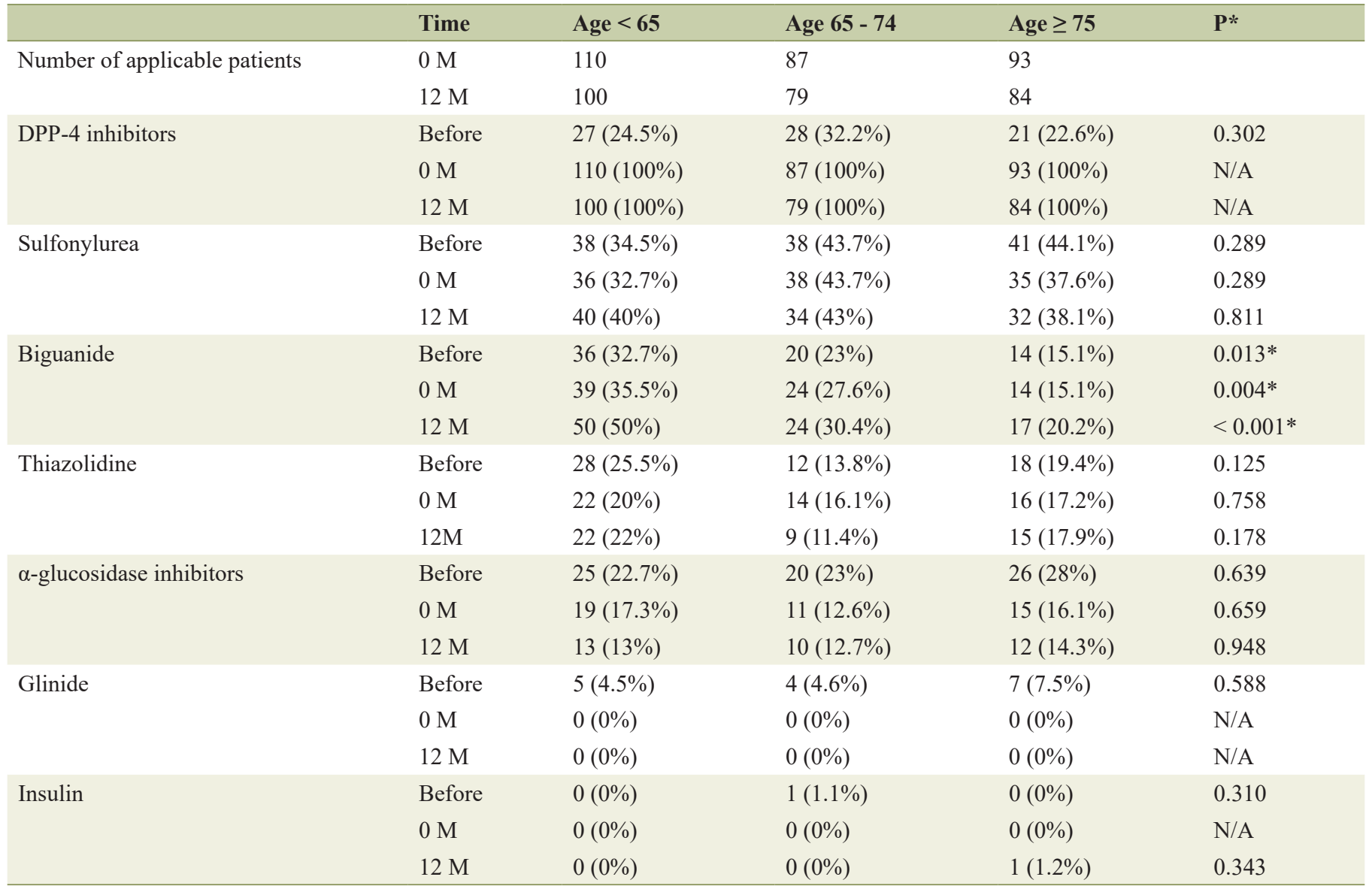

DPP-4: dipeptidyl peptidase-4; M: month; N/A: not applicable. ${ }^{*} \mathrm{P}<0.05$ Chi-square test.

cant difference is considered to have been a transient change. Our previous study of sitagliptin showed that eGFR decreased significantly in elderly subjects aged over 65; however, this parameter did not decrease in the patients with low baseline eGFR, although it decreased in the patients with high baseline eGFR [15]. Ujihara et al reported similar results [16]. Their study showed the eGFR change to be $-2.7 \pm 8.1$ in elderly patients receiving sitagliptin for 3 months, a level similar to ours in the present study; it can be conjectured that there is no major difference in renal effects in the elderly between sitagliptin and alogliptin. This can be supported by the fact that the renal excretion rate in healthy adults does not differ widely between
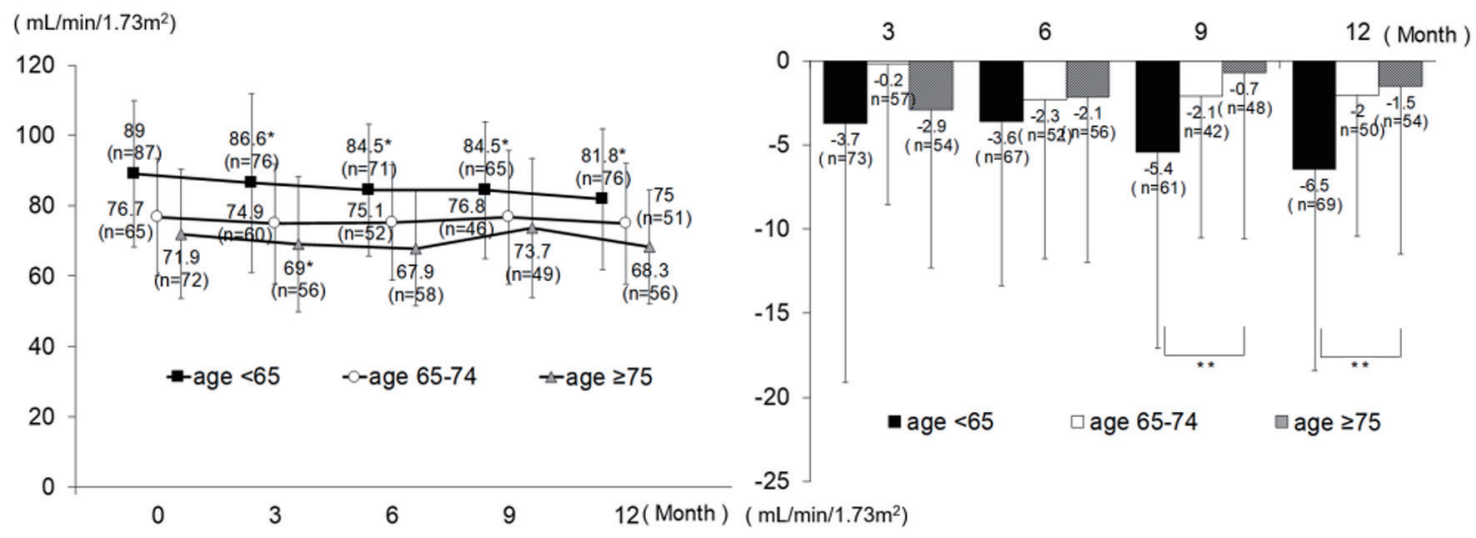

Figure 2. Changes in eGFR (all patients). ${ }^{*} \mathrm{P}<0.05$ versus 0 month, paired $t$-test; ${ }^{* *} \mathrm{P}<0.05$, ANOVA. eGFR: estimated glomerular filtration rate; ANOVA: analysis of variance. 


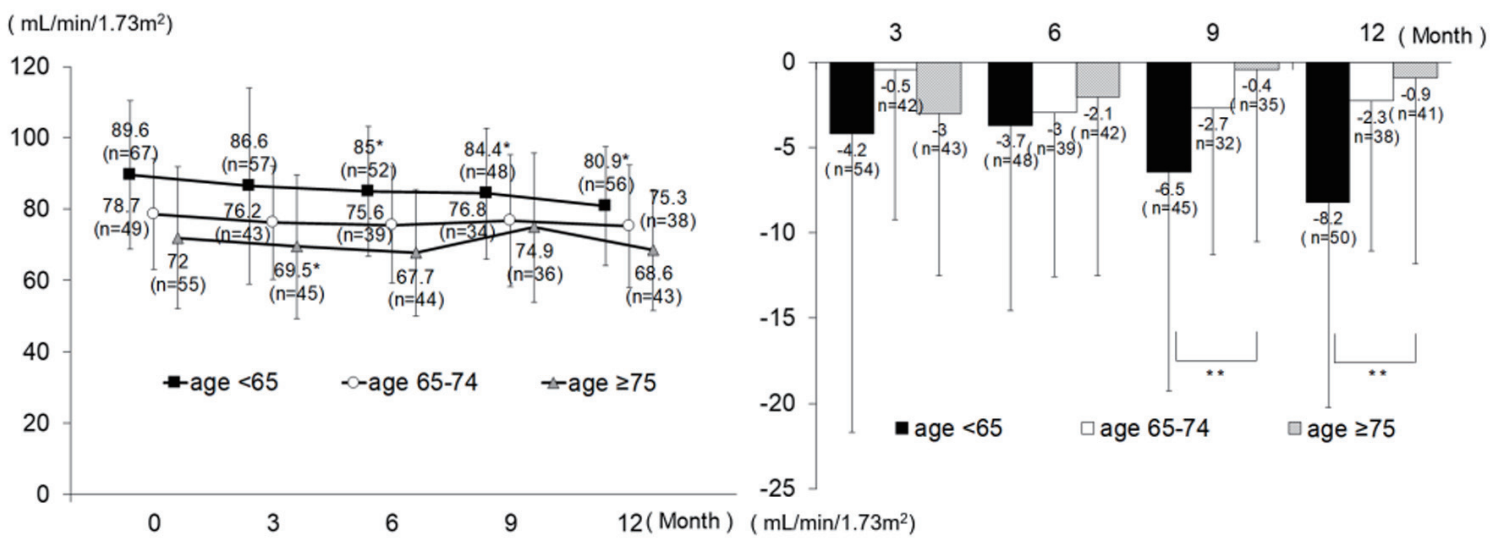

Figure 3. Changes in eGFR (patients newly receiving alogliptin). ${ }^{*} \mathrm{P}<0.05$ versus 0 month, paired $t$-test; ${ }^{* *} \mathrm{P}<0.05$, ANOVA. eGFR: estimated glomerular filtration rate; ANOVA: analysis of variance.

sitagliptin (87\%) [17] and alogliptin (76\%) [18].

HbA1c decreased significantly in all age groups. Our previous study of sitagliptin showed significant reductions in HbA1c irrespective of age group [15]; similar results were obtained with alogliptin in the present study. In terms of changes at month 3 , on the other hand, age-related differences were noted. We previously reported that the full-set analysis in the ATTAK-J study showed HbAlc to decrease with increasing initial $\mathrm{HbAlc}$ level and with decreasing duration of disease [5], and this may have influenced the results of the stratified analysis in the present study, in which the young subjects had higher HbAlc levels and shorter duration of disease. The age-stratified analysis in the present study showed satisfactory control of $\mathrm{HbAlc}$ to be $6.84 \pm 0.86$ at month 3 in the age group $\geq 75$; the study drug was not weakly effective in blood glucose reductions. DPP-4 inhibitors improve blood glucose control by increasing insulin secretion with a dependence on blood glucose level via inhibition of glucagon-like peptide-1 (GLP-1) inactivation and DPP-4-mediated action, thus inhibiting glucagon secretion $[19,20]$. This finding of HbAlc represents the characteristic dependence of the effects of the study drug on blood glucose. A meta-analysis of non-Japanese data by Pratley et al found a change in $\mathrm{HbAlc}$ of $-0.8 \%$ in the $\geq 65$ age group at month 26 of alogliptin treatment [13], which was greater than the $-0.5 \%$ change in the present study. This difference is attributable to the fact that the $8.0 \%$ initial $\mathrm{HbA} 1 \mathrm{c}$ level reported by Pratley et al [13] was higher than the baseline HbA1c levels of $7.06 \%$ to $7.39 \%$ in the elderly subjects in the present study.

In the elderly, kidney dysfunction can delay drug excretion and pose a hypoglycemia issue due to blood-glucose-lowering drugs. In the present study, hypoglycemia occurred at low incidences (i.e. in one patient in the age group $<65$ and one patient in the age group 65 - 74). This result demonstrates safety of alogliptin treatment in the elderly. One study reported, however, that severe hypoglycemia due to the use of a blood-glucoselowering agent was likely to occur in the elderly [21]. Patients at $\geq 75$ years of age often have disorders in cognitive and/or physical function [22-26], and their hypoglycemic symptoms are likely to be overlooked, which in turn can lead to symp- tom exacerbations [27]. Even with the use of DPP-4 inhibitors, which exhibit their effects with blood glucose dependence, it will continue to be necessary to encourage patients to be aware of their subjective symptoms of hypoglycemia to ensure that signs will not be overlooked.

No significant changes in BP were observed in any group, nor was there any intergroup difference. Although BW decreased significantly by $-0.3 \pm 1.5 \mathrm{~kg}$ at month 3 in the age group $\geq 75$, this decrease is considered to have no influence on the safety, since there was no significant difference at or after month 6 , and the changes were small and transient. The results for effects on lipid and liver function markers were similar to the previously reported full analysis results. Although subanalysis results revealed some tendencies not found in the full-set analysis results, the HDL-C reductions in the age group $65-74$ and GPT reductions in the age group $<65$ are attributable to higher baseline values of the respective parameters.

The results for the effects of alogliptin in elderly subjects in the present study showed tendencies similar to those for the effects of sitagliptin in elderly subjects in our previous study. Which of the two drugs is superior remains unknown, because of the absence of direct comparisons with a study population of elderly subjects only; however, it seems that there is no major difference in the efficacy or safety up to 1 year of treatment. Although many studies have compared the effects of different DPP-4 inhibitors in an analysis including young subjects, they reported variable results for blood glucose control effects and inconsistent judgments concerning the superiority of the drugs [28-35]. Meta-analyses have not found any significant difference in hypoglycemic action among different DPP-4 inhibitors $[36,37]$. In a meta-analysis focusing on alogliptin, Kay et al compared the effects of alogliptin with those of four other DPP-4 inhibitors (linagliptin, saxagliptin, sitagliptin, and vildagliptin) in patients with insufficient blood glucose control by treatment with metformin and sulfonylurea (SU), reporting that alogliptin did not differ from the other DPP-4 inhibitors in terms of efficacy and safety [38]. The excretory pathway differs among DPP-4 inhibitors. Alogliptin, sitagliptin, anagliptin, omarigliptin, and trelagliptin are excreted as unmetabolized drugs mainly from the kidney into the urine. Vildagliptin 


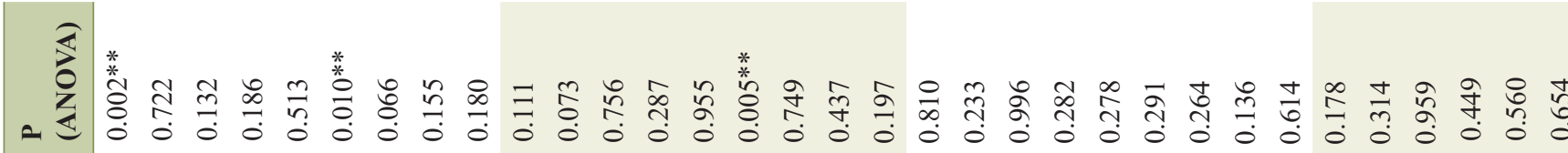

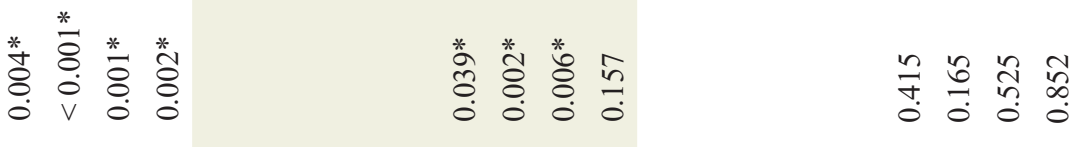

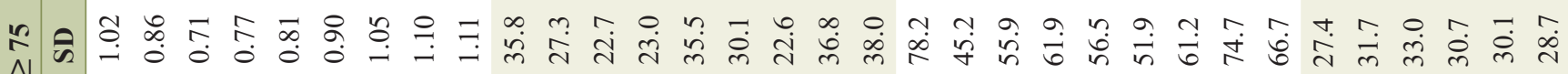
苔

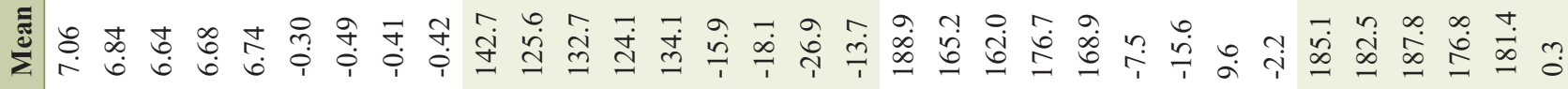

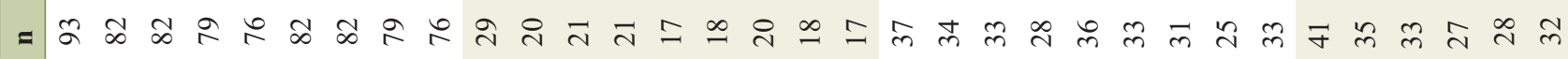

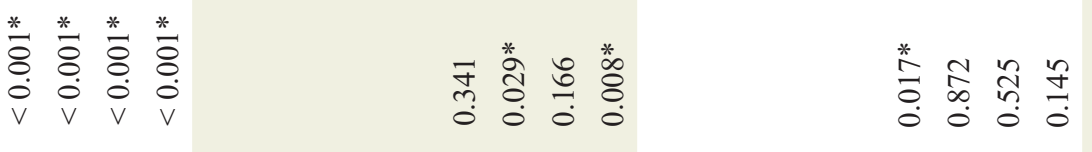

*

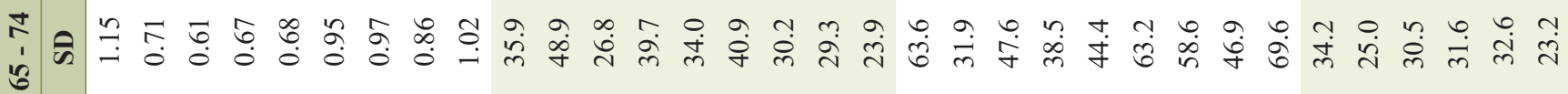
\&

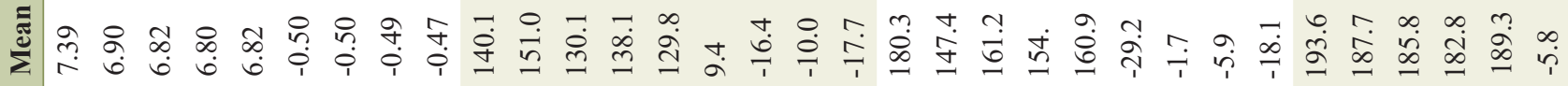

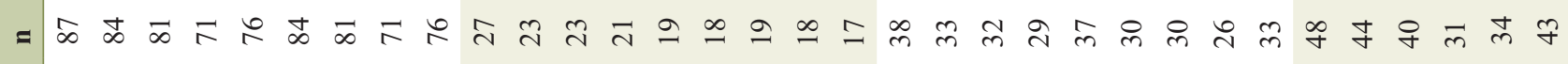

*.

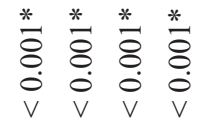

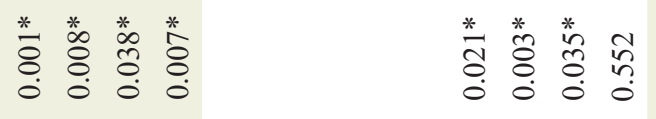

घ) 选

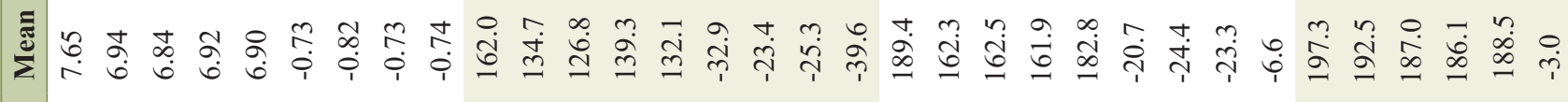

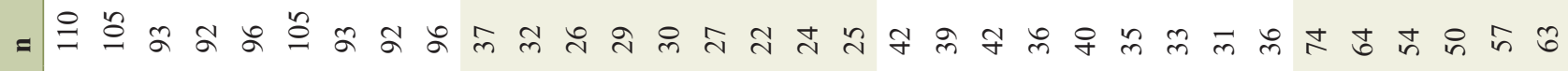

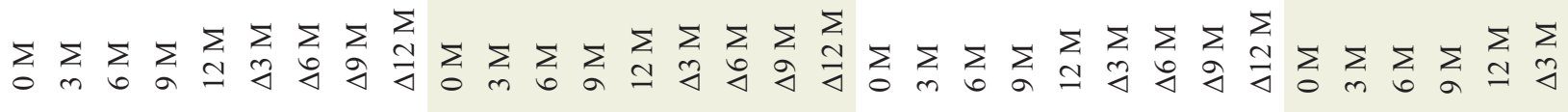

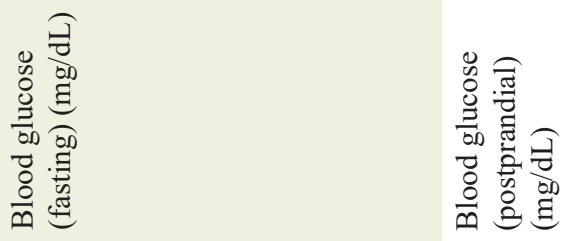

후일 


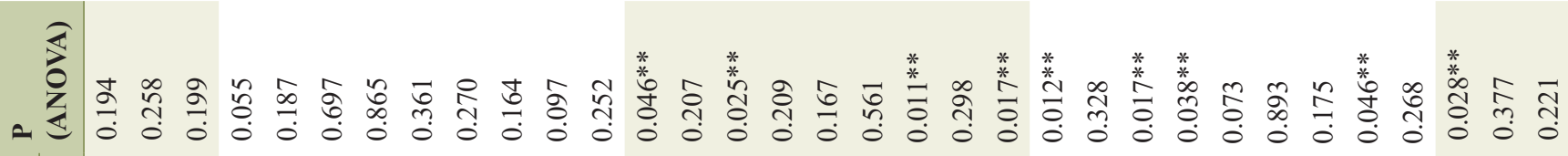

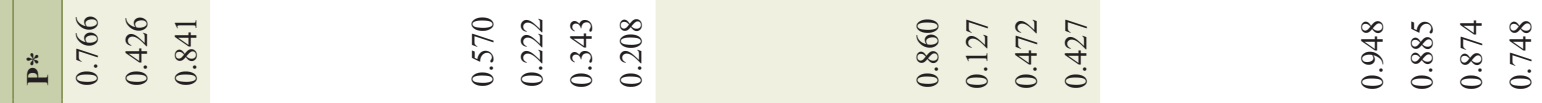

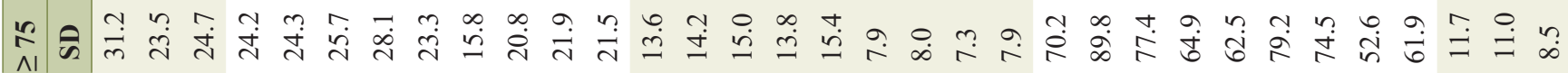
\&.0

馬

=

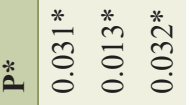

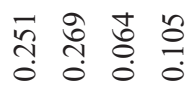

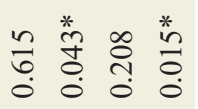

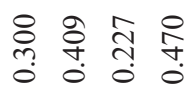

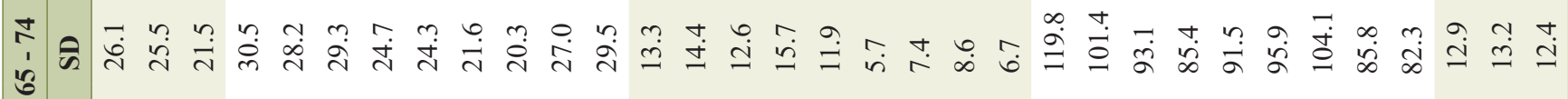
\&্口

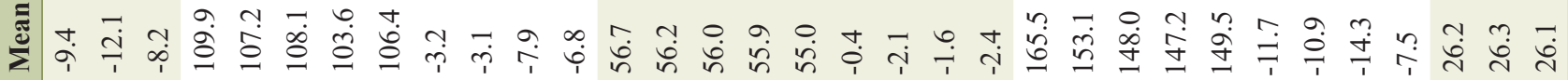

=

จิ

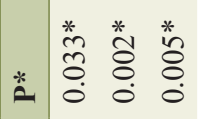

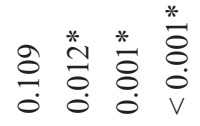

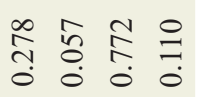

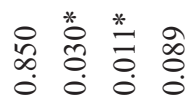

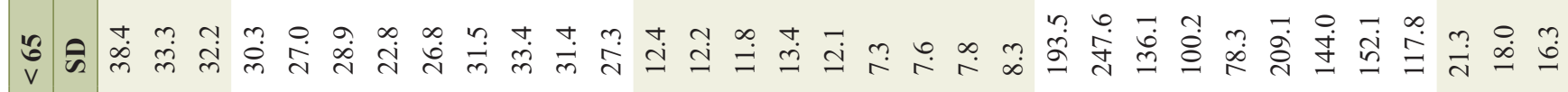
我

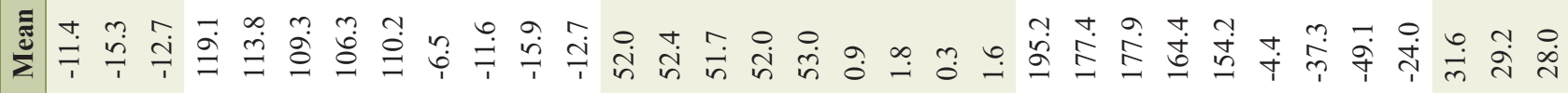

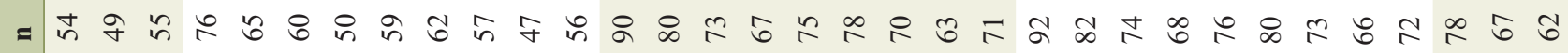

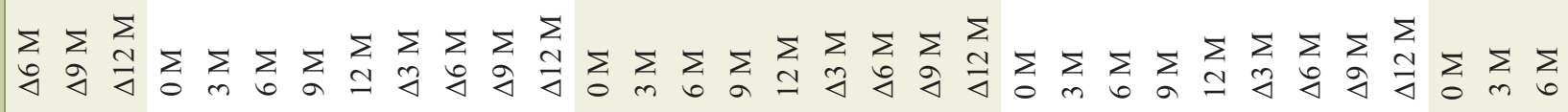

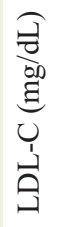

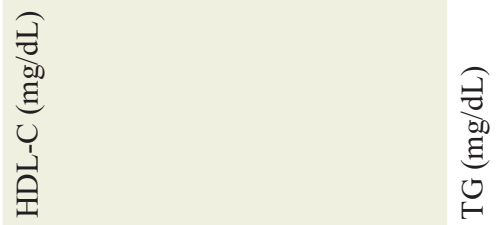

Ð 


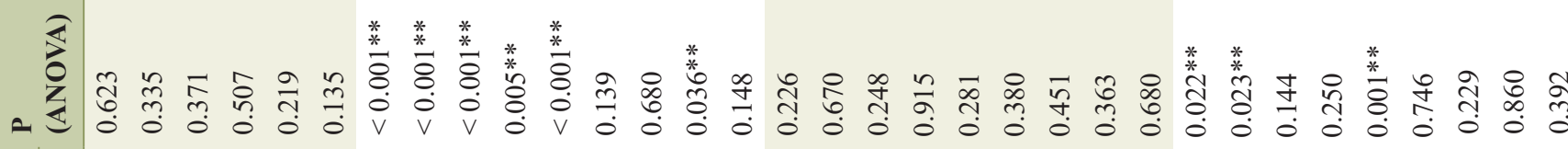

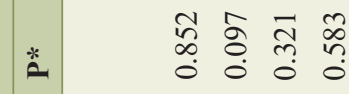
$\begin{array}{llll}0 & 0 & 0 & 0 \\ & 0 & 0 \\ 0 & 0 & 0 \\ 0 & 0\end{array}$

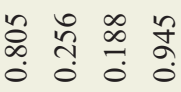

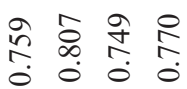

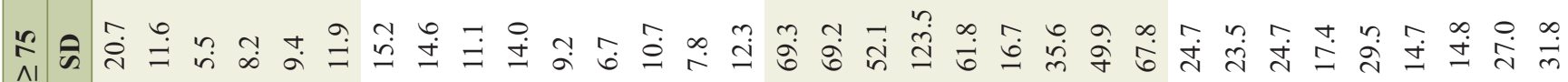

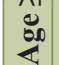

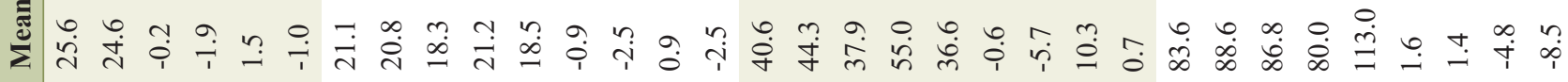

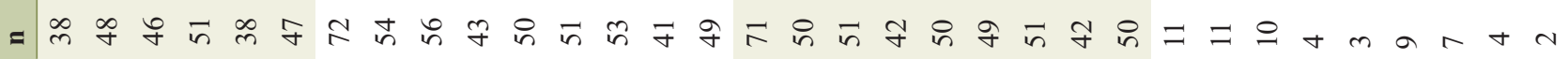

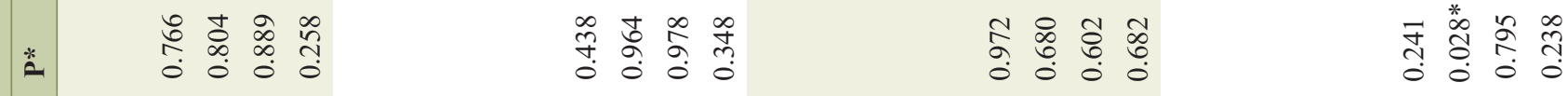

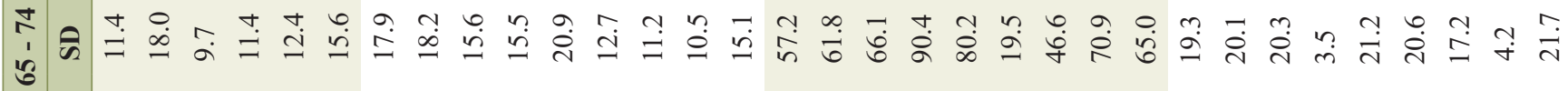
$\stackrel{8}{<}$

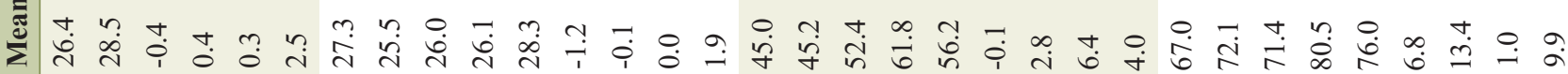

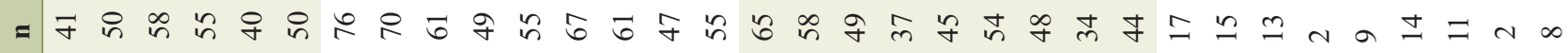

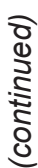

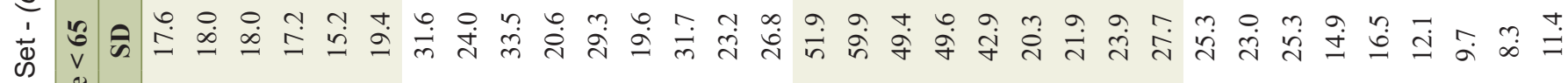
$\frac{\infty}{\infty} \stackrel{0}{\infty}$

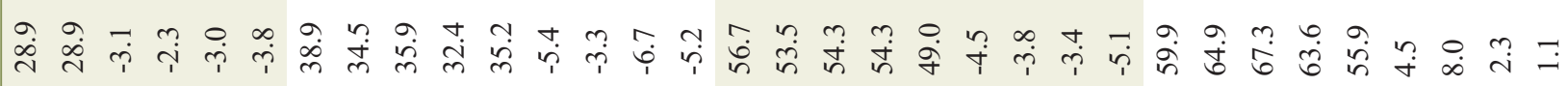
(S) 


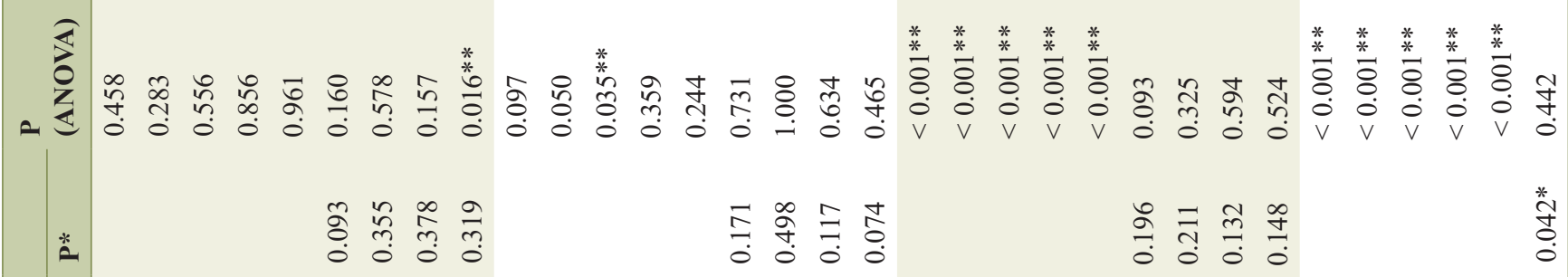

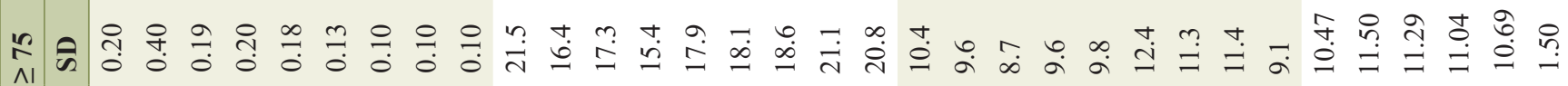
选

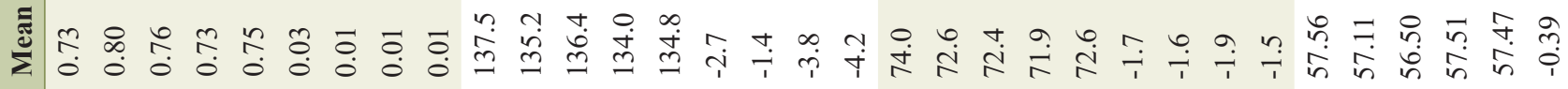

$=\mathbb{1}$ i

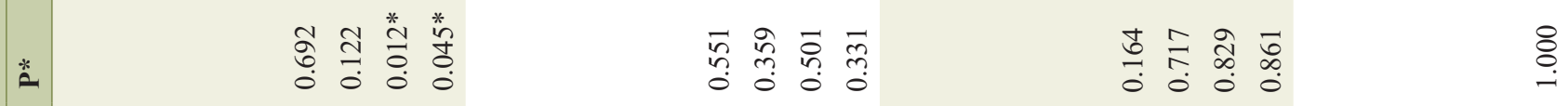

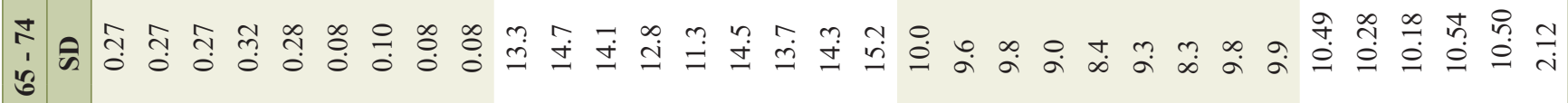
\&

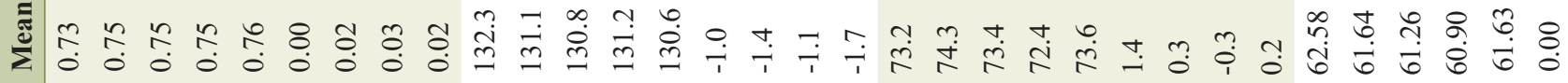

$=\curvearrowleft 8$ ำ

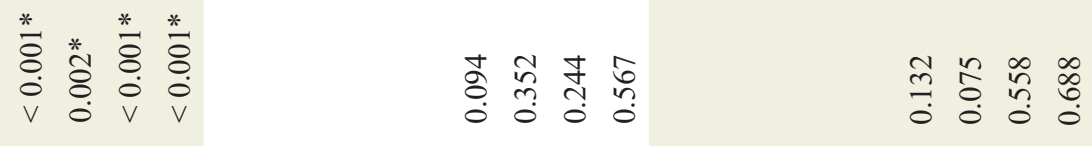

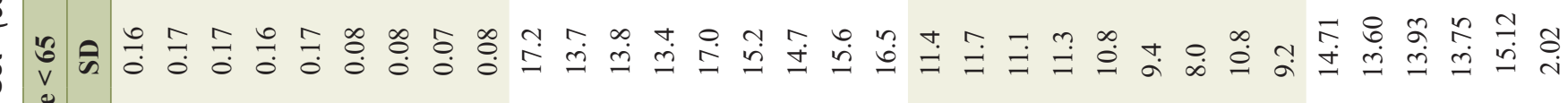

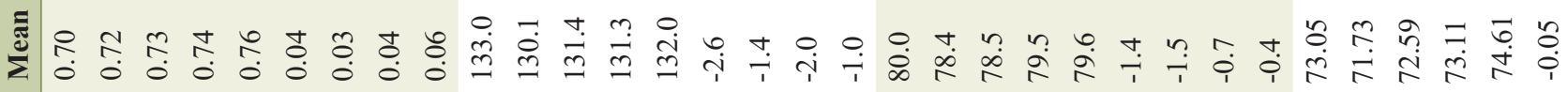

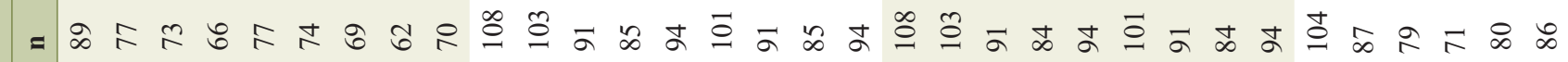

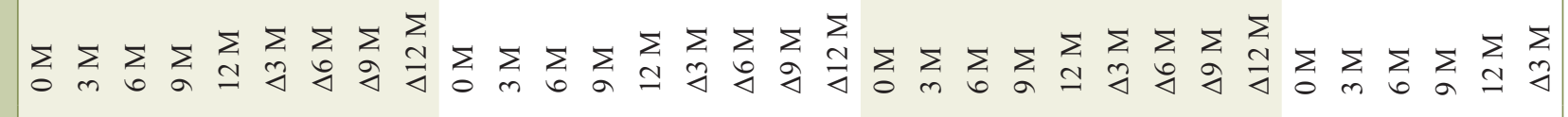

总 


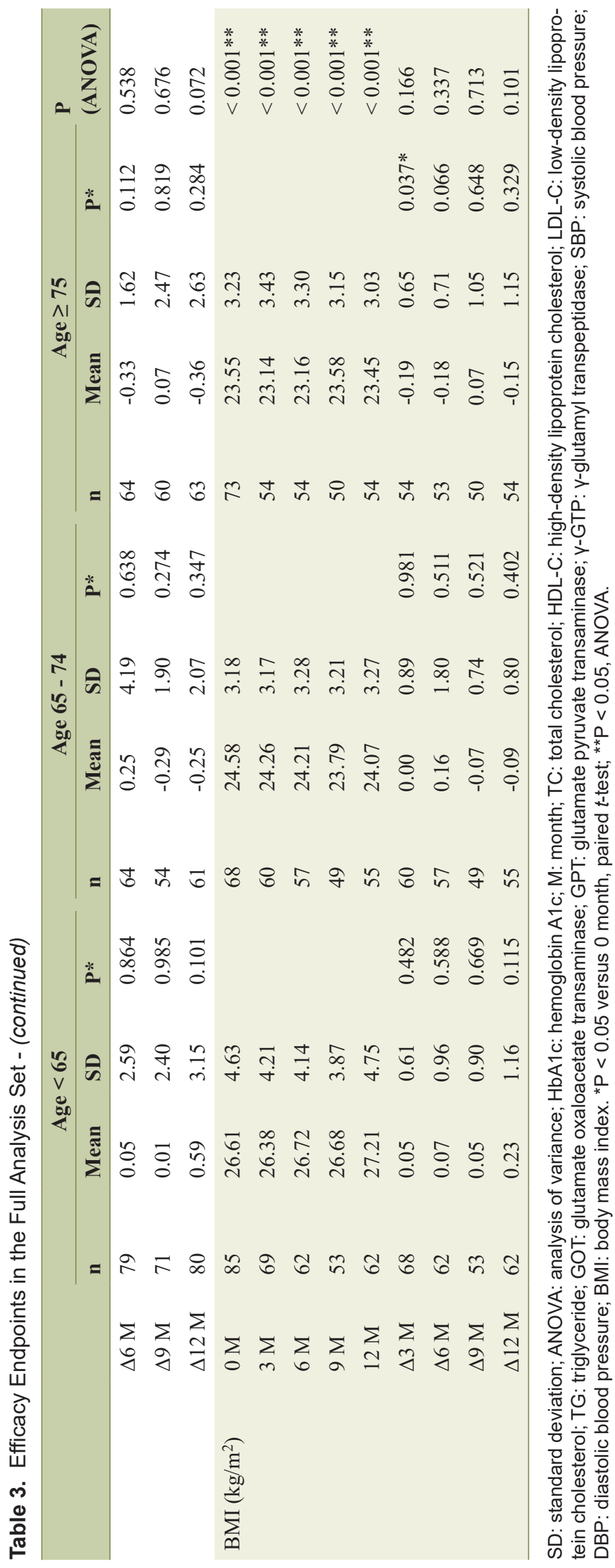

and saxagliptin are metabolized mainly in the liver. Linagliptin is excreted from the biliary system, and teneligliptin is metabolized in the liver and excreted from the renal system. In the elderly, in particular, the excretory pathways for these drugs can influence their safety; therefore, further evidence must be compiled.

As stated in the Introduction, Japan is facing an issue of preventing initiation of hemodialysis due to exacerbation of diabetic nephropathy. In October 2017, the Japanese Society of Nephrology and Japan Diabetes Society jointly adopted the Strategic Action Plan for Diabetic Kidney Disease (DKD) [39]. Since that time, these groups have been collaborating on implementation of DKD status surveys, elucidating its pathology, and developing new treatments. The present study showed alogliptin to be effective and safe in the treatment of elderly patients, providing valuable therapeutic evidence for elderly people as incipient diabetics. Generally, clinical trials include relatively small proportions of elderly subjects both in and outside Japan; data obtained for elderly subjects prior to the approval of the study drug can be insufficient. Conducted as a survey in actual clinical settings, unlike clinical trials, the present study is of great clinical implication by providing such data as valuable evidence for diabetic treatment.

\section{Limitations}

The present study lacked a control group, because it was conducted in an open-label retrospective fashion. In addition, no data on concomitant use with sodium-glucose transport protein 2 (SGLT2) inhibitors were available, because they were not approved at the time the data were obtained. Patients with GLP-1 receptor agonists were not included in this study. There are two backgrounds. One is that we acquired retrospective data at that time when DPP-4 inhibitors were approved. Another is that in our study subject, DPP-4 inhibitors were better suited for patients than GLP-1 analogues. The study was not based on accurate power calculations, because of its nature as an exploratory analysis.

\section{Conclusions}

Alogliptin significantly lowers $\mathrm{HbA} 1 \mathrm{c}$ levels in the elderly and can be used without posing any safety issues, including renal effects, thus contributing to safe blood glucose control in clinical practice.

\section{Acknowledgments}

The ATTAK-J study was performed by the Diabetes Committee Study Group of the Kanagawa Physicians Association. We thank the doctors and staff of the Kanagawa Physicians Association for cooperating with this research, and the patients for their participation in the study. The following hospitals/ clinics and investigators from the Kanagawa Physicians Association participated in this study: Sakura T's Clinic (Dr. 
Toshiaki Sakurazawa); Sakurai Clinic (Dr. Atsuko Sakurai); Mineki Naika Clinic (Dr. Hitoshi Mineki); Nippon Yakin Kogyo Clinic (Dr. Mitsuyuki Taira); Hayashi Diabetic Medicine Clinic (Dr. Tsutomu Hayashi); Machida Gastroenterology and Surgery Clinic (Dr. Tomoyuki Machida); Hara Clinic (Dr. Yoshikuni Hara); Shonan Chuo Clinic (Dr. Atsunori Oishi); AikawaHokubu Hospital (Dr. Hironori Adachi); Kubota Clinic (Dr. Takahiro Kubota); Hashimoto Clinic (Dr. Kenji Sakurai); Minami-guchi Clinic, Kuzuhadai Hospital (Dr. Takayuki Furuki); Suzuki Clinic (Dr. Shin Suzuki); Kikuchi Surgery and Internal Medicine Clinic (Dr. Hiroshi Kikuchi).

\section{Financial Disclosure}

This study was funded by the Japan Diabetes Foundation.

\section{Conflict of Interest}

This study was designed and planned by members of the Kanagawa Physicians Association and was funded by the Japan Diabetes Foundation. No company was involved in designing the study, patient enrollment, calculation or analysis of the data, interpretation of the findings, or writing the manuscript.

\section{Informed Consent}

Before participation, all patients provided written informed consent.

\section{Author Contributions}

IM conceived and designed the experiments and analyzed the data; HT, NS, SI, MO, TT, MT, HK, HM, AK and IM enrolled patients; HT and IM wrote the first draft of the manuscript and contributed to the writing of the manuscript; HT, NS, SI, MO, TT, MT, HK, HM, AK and IM agreed with the manuscript's results and conclusions; All authors have read, and confirmed that they meet ICMJE criteria for authorship.

\section{References}

1. The Japanese Society for Dialysis Therapy. Current status of dialysis therapy. 2017. Retrieved from: https://docs. jsdt.or.jp/overview/. Accessed 7 July 2019.

2. Choudhury D, Ahmed Z. Drug-associated renal dysfunction and injury. Nat Clin Pract Nephrol. 2006;2(2):80-91.

3. Scheen AJ. A review of gliptins for 2014. Expert Opin Pharmacother. 2015;16(1):43-62.

4. Scheen AJ. Safety of dipeptidyl peptidase-4 inhibitors for treating type 2 diabetes. Expert Opin Drug Saf. 2015;14(4):505-524.

5. Takeda H, Sasai N, Ito S, Obana M, Takuma T, Takai M,
Kaneshige H, et al. Efficacy and Safety of Alogliptin in Patients With Type 2 Diabetes: Analysis of the ATTAK-J Study. J Clin Med Res. 2016;8(2):130-140.

6. White WB, Cannon CP, Heller SR, Nissen SE, Bergenstal RM, Bakris GL, Perez AT, et al. Alogliptin after acute coronary syndrome in patients with type 2 diabetes. N Engl J Med. 2013;369(14):1327-1335.

7. White WB, Heller SR, Cannon CP, Howitt H, Khunti K, Bergenstal RM, Investigators E. Alogliptin in Patients with Type 2 Diabetes Receiving Metformin and Sulfonylurea Therapies in the EXAMINE Trial. Am J Med. 2018;131(7):813-819 e815.

8. Saisho Y. Alogliptin benzoate for management of type 2 diabetes. Vasc Health Risk Manag. 2015;11:229-243.

9. Seino Y, Fujita T, Hiroi S, Hirayama M, Kaku K. Efficacy and safety of alogliptin in Japanese patients with type 2 diabetes mellitus: a randomized, double-blind, dose-ranging comparison with placebo, followed by a long-term extension study. Curr Med Res Opin. 2011;27(9):17811792.

10. Kaku K, Mori M, Kanoo T, Katou M, Seino Y. Efficacy and safety of alogliptin added to insulin in Japanese patients with type 2 diabetes: a randomized, double-blind, 12-week, placebo-controlled trial followed by an openlabel, long-term extension phase. Expert Opin Pharmacother. 2014;15(15):2121-2130.

11. Mita T, Katakami N, Yoshii H, Onuma T, Kaneto H, Osonoi T, Shiraiwa T, et al. Erratum. Alogliptin, a Dipeptidyl Peptidase 4 Inhibitor, Prevents the Progression of Carotid Atherosclerosis in Patients With Type 2 Diabetes: The Study of Preventive Effects of Alogliptin on Diabetic Atherosclerosis (SPEAD-A). Diabetes Care 2016;39:139148. Diabetes Care. 2017;40(6):808

12. Takebayashi K, Suzuki T, Naruse R, Hara K, Suetsugu M, Tsuchiya T, Inukai T. Long-Term Effect of Alogliptin on Glycemic Control in Japanese Patients With Type 2 Diabetes: A 3.5-Year Observational Study. J Clin Med Res. 2017;9(9):802-808.

13. Pratley RE, McCall T, Fleck PR, Wilson CA, Mekki Q. Alogliptin use in elderly people: a pooled analysis from phase 2 and 3 studies. J Am Geriatr Soc. 2009;57(11):2011-2019.

14. Rosenstock J, Wilson C, Fleck P. Alogliptin versus glipizide monotherapy in elderly type 2 diabetes mellitus patients with mild hyperglycaemia: a prospective, doubleblind, randomized, 1-year study. Diabetes Obes Metab. 2013;15(10):906-914.

15. Umezawa S, Kubota A, Maeda H, Kanamori A, Matoba $\mathrm{K}$, Jin Y, Minagawa F, et al. Two-year assessment of the efficacy and safety of sitagliptin in elderly patients with type 2 diabetes: Post hoc analysis of the ASSET-K study. BMC Endocr Disord. 2015;15:34.

16. Ujihara N, Sakura H, Hashimoto N, Sasamoto K, Ohashi H, Hasumi S, Kasahara T, et al. Erratum to: Efficacy and safety of sitagliptin in elderly patients with type 2 diabetes mellitus and comparison of hypoglycemic action of concomitant medications: a subanalysis of the JAMP study. Diabetol Int. 2018;9(1):66-67.

17. Vincent SH, Reed JR, Bergman AJ, Elmore CS, Zhu B, 
Xu S, Ebel D, et al. Metabolism and excretion of the dipeptidyl peptidase 4 inhibitor [14C] sitagliptin in humans. Drug Metab Dispos. 2007;35(4):533-538.

18. Capuano A, Sportiello L, Maiorino MI, Rossi F, Giugliano D, Esposito K. Dipeptidyl peptidase-4 inhibitors in type 2 diabetes therapy - focus on alogliptin. Drug Des Devel Ther. 2013;7:989-1001.

19. Drucker DJ. Enhancing incretin action for the treatment of type 2 diabetes. Diabetes Care. 2003;26(10):29292940.

20. Herman GA, Bergman A, Stevens C, Kotey P, Yi B, Zhao $\mathrm{P}$, Dietrich B, et al. Effect of single oral doses of sitagliptin, a dipeptidyl peptidase-4 inhibitor, on incretin and plasma glucose levels after an oral glucose tolerance test in patients with type 2 diabetes. J Clin Endocrinol Metab. 2006;91(11):4612-4619.

21. Seltzer HS. Drug-induced hypoglycemia. A review of 1418 cases. Endocrinol Metab Clin North Am. 1989;18(1):163-183.

22. Araki A. Low well-being, cognitive impairment and visual impairment associated with functional disabilities in elderly Japanese patients with diabetes mellitus. Geriatrics \& Gerontology International. 2004;4(1):15-24.

23. Ma F, Wu T, Miao R, Xiao YY, Zhang W, Huang G. Conversion of mild cognitive impairment to dementia among subjects with diabetes: a population-based study of incidence and risk factors with five years of follow-up. J Alzheimers Dis. 2015;43(4):1441-1449.

24. Solini A, Penno G, Bonora E, Fondelli C, Orsi E, Trevisan $\mathrm{R}$, Vedovato $\mathrm{M}$, et al. Age, renal dysfunction, cardiovascular disease, and antihyperglycemic treatment in type 2 diabetes mellitus: findings from the Renal Insufficiency and Cardiovascular Events Italian Multicenter Study. J Am Geriatr Soc. 2013;61(8):1253-1261.

25. Huang ES, Liu JY, Moffet HH, John PM, Karter AJ. Glycemic control, complications, and death in older diabetic patients: the diabetes and aging study. Diabetes Care. 2011;34(6):1329-1336.

26. Lind M, Olsson M, Rosengren A, Svensson AM, Bounias I, Gudbjornsdottir S. The relationship between glycaemic control and heart failure in 83,021 patients with type 2 diabetes. Diabetologia. 2012;55(11):2946-2953.

27. Bremer JP, Jauch-Chara K, Hallschmid M, Schmid S, Schultes B. Hypoglycemia unawareness in older compared with middle-aged patients with type 2 diabetes. Diabetes Care. 2009;32(8):1513-1517.

28. Sakamoto M, Nishimura R, Irako T, Tsujino D, Ando K, Utsunomiya K. Comparison of vildagliptin twice daily vs. sitagliptin once daily using continuous glucose monitoring (CGM): crossover pilot study (J-VICTORIA study). Cardiovasc Diabetol. 2012;11:92.
29. Marfella R, Barbieri M, Grella R, Rizzo MR, Nicoletti GF, Paolisso G. Effects of vildagliptin twice daily vs. sitagliptin once daily on 24-hour acute glucose fluctuations. J Diabetes Complications. 2010;24(2):79-83.

30. Guerci B, Monnier L, Serusclat P, Petit C, Valensi P, Huet D, Raccah D, et al. Continuous glucose profiles with vildagliptin versus sitagliptin in add-on to metformin: results from the randomized Optima study. Diabetes Metab. 2012;38(4):359-366.

31. Tang YZ, Wang G, Jiang ZH, Yan TT, Chen YJ, Yang M, Meng LL, et al. Efficacy and safety of vildagliptin, sitagliptin, and linagliptin as add-on therapy in Chinese patients with T2DM inadequately controlled with dual combination of insulin and traditional oral hypoglycemic agent. Diabetol Metab Syndr. 2015;7:91.

32. Kanozawa K, Noguchi Y, Sugahara S, Nakamura S, Yamamoto H, Kaneko K, Kono R, et al. The renoprotective effect and safety of a DPP-4 inhibitor, sitagliptin, at a small dose in type 2 diabetic patients with a renal dysfunction when changed from other DPP-4 inhibitors: REAL trial. Clin Exp Nephrol. 2018;22(4):825-834.

33. Shigematsu E, Yamakawa T, Oba MS, Suzuki J, Nagakura J, Kadonosono K, Terauchi Y. A Randomized Controlled Trial of Vildagliptin Versus Alogliptin: Effective Switch From Sitagliptin in Patients With Type 2 Diabetes. J Clin Med Res. 2017;9(7):567-572.

34. Tanaka K, Okada Y, Mori H, Miyazaki M, Kuno F, Sonoda S, Sugai K, et al. Comparative analysis of the effects of alogliptin and vildagliptin on glucose metabolism in type 2 diabetes mellitus. Endocr J. 2017;64(2):179-189.

35. Kurozumi A, Okada Y, Arao T, Kobayashi T, Masuda D, Yamashita S, Tanaka Y. Comparison of effects of anagliptin and alogliptin on serum lipid profile in type 2 diabetes mellitus patients. J Diabetes Investig. 2018;9(2):360-365.

36. Aroda VR, Henry RR, Han J, Huang W, DeYoung MB, Darsow T, Hoogwerf BJ. Efficacy of GLP-1 receptor agonists and DPP-4 inhibitors: meta-analysis and systematic review. Clin Ther. 2012;34(6):1247-1258 e1222.

37. Craddy P, Palin HJ, Johnson KI. Comparative effectiveness of dipeptidylpeptidase-4 inhibitors in type 2 diabetes: a systematic review and mixed treatment comparison. Diabetes Ther. 2014;5(1):1-41.

38. Kay S, Strickson A, Puelles J, Selby R, Benson E, Tolley K. Comparative effectiveness of adding alogliptin to metformin plus sulfonylurea with other DPP-4 inhibitors in type 2 diabetes: a systematic review and network metaanalysis. Diabetes Ther. 2017;8(2):251-273.

39. Kadowaki T, Kashihara N. Strategic action plan for diabetic kidney disease. Japanese Society of Nephrology and the Japan Diabetes Society. 2017. Retrieved from https:// cdn.jsn.or.jp/data/STOP-DKD.pdf. Accessed 7 July 2019. 